Kooperation als Strategie ökologischer Unternehmenspolitik

\title{
Die Vielfalt der Möglichkeiten
}

\author{
Kooperationen, die so selbstverständlich in die Diskussion um ökologische \\ Unternehmenspolitik eingebracht werden, sind in mehrfacher Hinsicht wenig \\ eindeutig. Fußen Kooperationen auf individuellen Strategien oder kollektiven \\ Normen? Sind sie gebunden an den Dialog? Welche Rolle spielt Vertrauen? \\ Was unterscheidet Kooperationen von Netzwerken?
}

\begin{abstract}
A Von Andreas Aulinger uf der Suche nach Möglichkeiten zur Beendigung der gegenwärtigen ökologischen Veränderungen der Welt werden im Bereich der Ökonomie nicht mehr nur ordnungspolitische Lösungen oder Lösungen innerhalb einzelner Unternehmen diskutiert, sondernd zunehmend Kooperationslösungen. Mit diesen sollen die Mängel der ersteren überwunden werden, nämlich:
\end{abstract}

- zu starke Eingriffe in die Autonomie der Unternehmen bei ordnungspolitischen Lösungen und

- zu geringe Tragweite im Verhältnis zu den anstehenden Problemen bei Lösungen innerhalb einzelner Unternehmen. Hinzu kommt hier, daß Unternehmen, die dennoch sehr weitreichende ökologische Umorientierungen vornehmen, Gefahr laufen, dies am Markt nicht honoriert zu bekommen.

Mit Kooperationslösungen soll die Handlungsautonomie für ökologisch notwendige Anpassungen nicht dem Gesetzgeber übertragen werden, sondern auf der Seite der Unternehmen bestehen bleiben. Dennoch werden hier Handlungsfelder eröffnet, die weit über den Möglichkeiten des einzelnen Unternehmens liegen. Das damit angespro-chene Kooperationsprinzip wird jedoch fast automatisch auf branchenweite Koope-rationen im Sinne von Selbstverpflichtungs-abkommen begrenzt. Selbstverpflichtungsabkommen stellen jedoch nur eine von vielen denkbaren Kooperationsformen zwischen Unternehmen dar. Bevor diese Vielfalt dargestellt wird, seien zunächst einige grundsätzliche Überlegungen zum Phänomen der Kooperation gestattet:

\section{Was ist eigentlich Kooperation?}

Zur Beantwortung dieser Frage hilft zunächst ein Ausflug in die bereits etwas ältere Literatur der Soziologie. Nach Deutsch ist eine Koopera- tionssituation dadurch gekennzeichnet, daß jeder an dieser Situation Beteiligte seine Ziele nur in dem Maße erreichen kann, wie auch die anderen Beteiligten ihre Ziele erreichen (1). Dabei ist keineswegs erforderlich, daß die Beteiligten identische Ziele haben. Es genügt, daß vorhandene Ziele miteinander harmonieren, um den Boden für eine Kooperation zu schaffen. Wenn dagegen einzelne Akteure ihre Ziele nur erreichen können, wenn die anderen ihre Ziele nicht erreichen, handelt es sich um eine Konkurrenzsituation. Zu dieser Definition von Kooperation (und Konkurrenz) ist nun zunächst einmal sehr Ernüchterndes zu sagen:

(1) Sie ist nicht die einzig denkbare.

(2) Nach ihr sind auch klassische Kaufverträge Kooperationen, $\mathrm{zu}$ denen doch gerade eine Abgrenzung geschaffen werden soll. (Niemand wïrde in einen Kaufvertrag einwilligen, wenn er damit nicht bestimmte Ziele zu erreichen gedenkt.)
Wenn diese Definition hier dennoch nicht nur erwähnt, sondern auch an ihr festgehalten wird, so bedarf dies einiger Erläuterungen:

Ad 1: Die Definition von Deutsch basiert auf der Existenz individueller, aber harmonierender Ziele der Kooperationspartner. Daneben gibt es Definitionsansätze, bei denen die Verständigungsorientierung der Kooperationspartner im Mittelpunkt steht. Beispielhaft sei hier auf Biesecker hingewiesen, die die Bedeutung von Diskursen als Elemente der Kooperation hervorhebt: „Dazu ist eine Form des Handelns nötig, die mehr einschließt als die eigennützige Maximierung des Handlungserfolgs, eine Form des Handelns, die neben dem Erfolg die Verständigung zwischen den KooperationspartnerInnen selbst zum Ziel hat" (2). Neben den eher ,strategischen Kooperationen“, wie sie sich aus der Definition von Deutsch ergeben, können danach auch „verständige Kooperationen“ beschrieben werden. Bei diesen stehen Diskurse und Prinzipien der Fairneß und Gegenseitigkeit im Vordergrund, die in der Anerkennung der anderen als gleichberechtigt und gleichgewichtig zum Ausdruck kommen.

Obwohl ich diese alternative Interpretation der Kooperation für sehr wichtig halte, soll hier für eine grundlegende Begriffsbestimmung der Kooperation die Existenz individueller Ziele nicht nur geduldet, sondern als konstitutives Element von Kooperationen betrachtet werden. Mit einem solchen Grundverständnis habe ich

\section{Abb.1: Morphologischer Kasten zur Darstellung der verschiedenen Kooperationsformen}

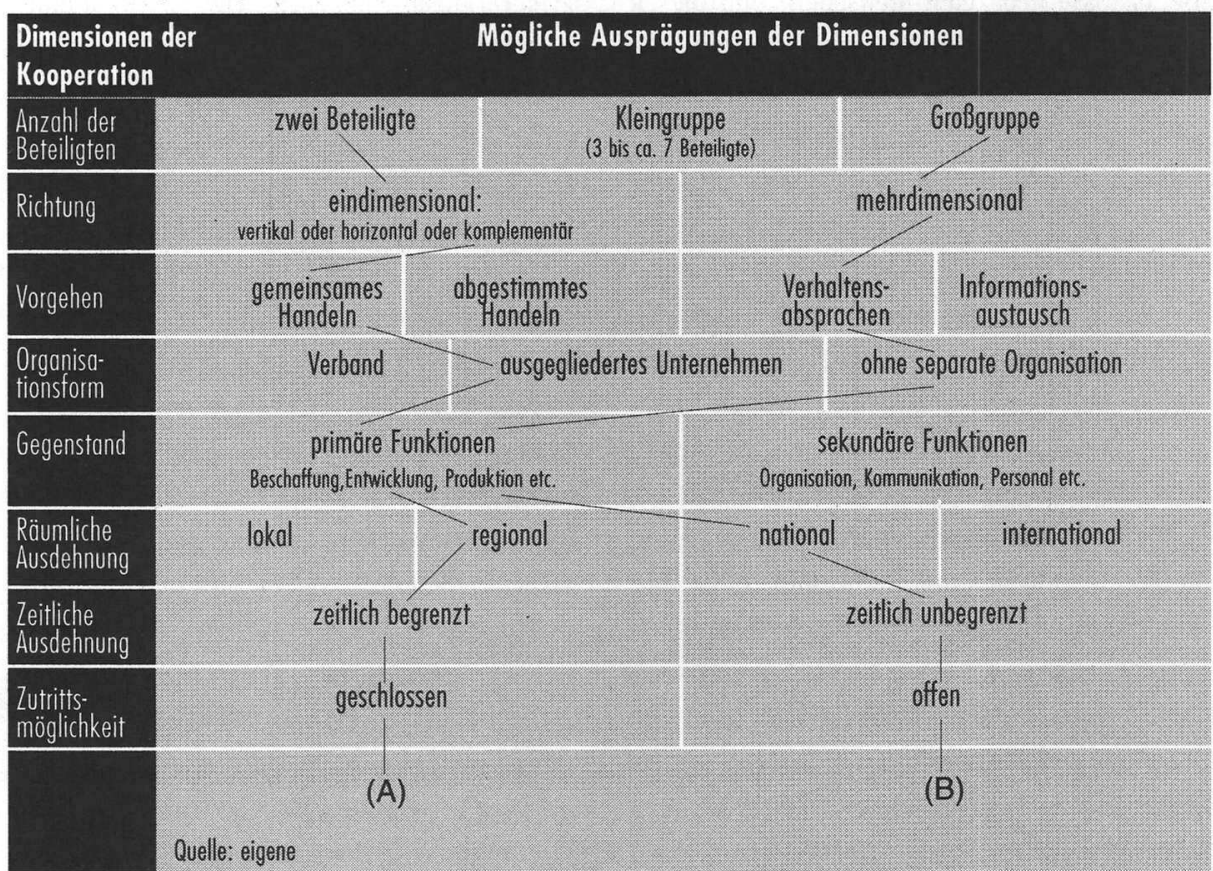


Abb. 2.1: Aus der Überlagerung von Unternehmenskooperationen ...

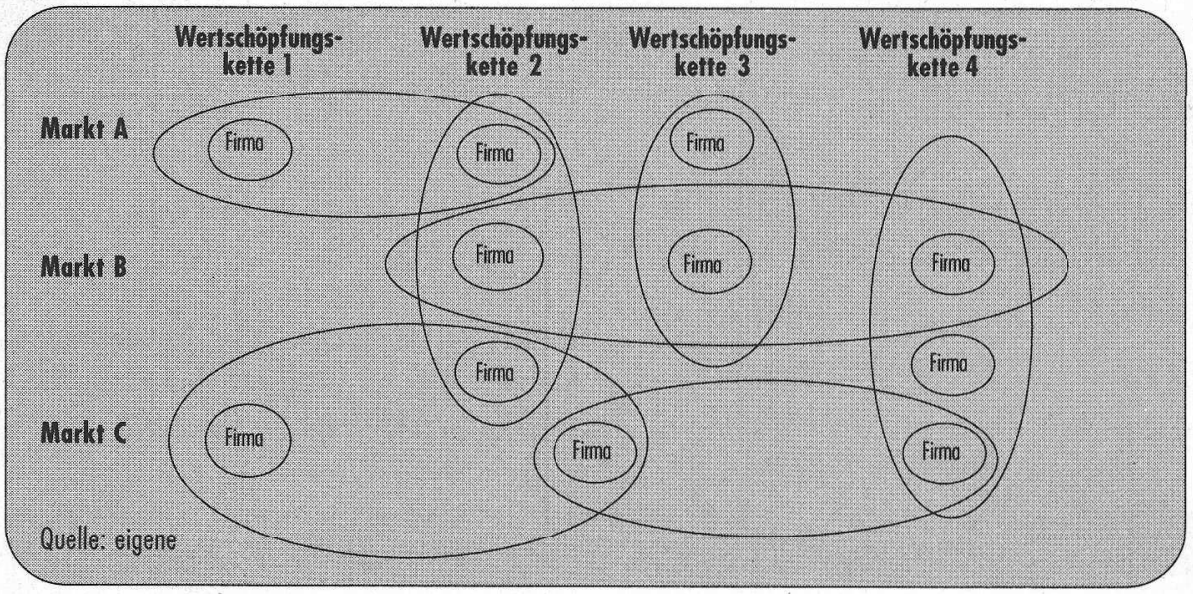

Abb. 2.2: ... entstehen Unternehmensnetzwerke

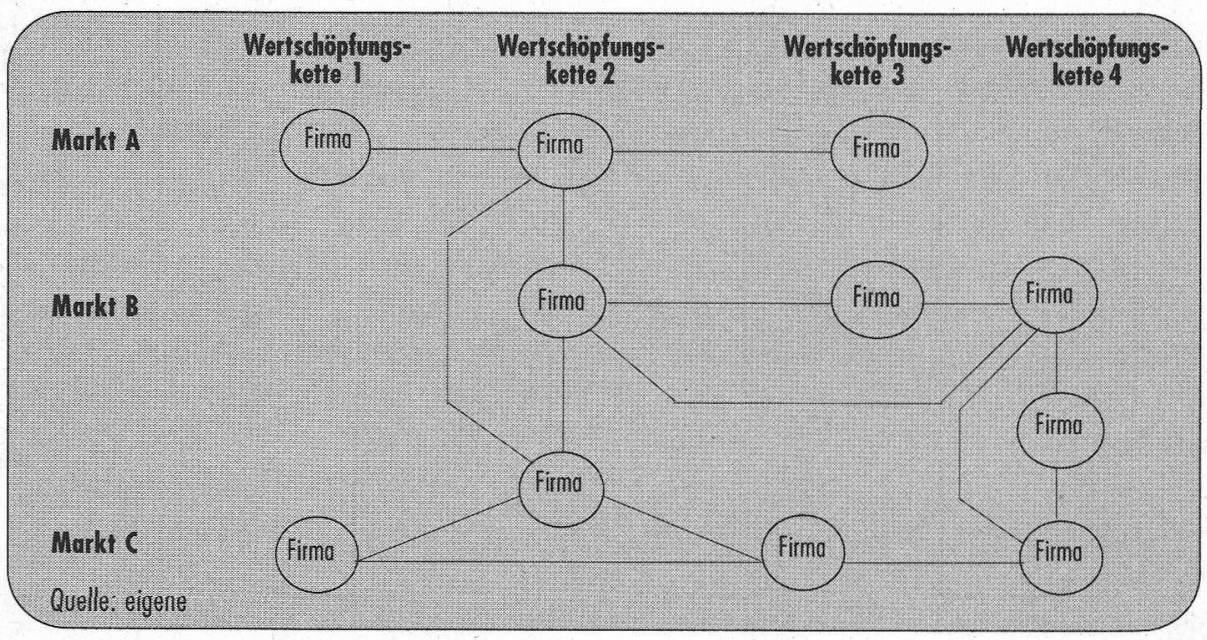

keineswegs den homo öconomicus als tumben Nutzenmaximierer und Kooperationspartner vor Augen. Es geht mir vielmehr um den von komplexen Überlagerungen verschiedenster Bedürfnisse geprägten Menschen. Und diesen Menschen möchte ich bei der Definition von Kooperationen nicht aus dem Auge verlieren. Deshalb halte ich es für hilfreich, die Existenz individueller Ziele und den Wunsch, diese zu verfolgen, als explizite Merkmale von Kooperationen zu begreifen. Dies schließt aber keineswegs aus, daß Kooperationen auf der Basis von Kommunikation oder verständigungsorientierten Diskursen erfolgen können. Beides kann sogar Voraussetzung dafür sein, sich über die Existenz verschiedener Ziele auszutauschen und Zielharmonien sichtbar zu machen. Kooperationen werden hier aber nicht mit Kommunikation oder bestimmten Diskursformen gleichgesetzt.

Ad 2: Miteinander harmonierende Ziele verschiedener Individuen sind damit aus meiner Sicht zwar ein unverzichtbares, aber noch kein hinreichendes Kriterium, um Kooperationen im en aber nicht mur insof Kooperationspartner werde seinen Kooperationsheitrag leisten. Sondern Vertrauen inshesondere dadurch, daß man sich selbst bindet und seinen eigenen Beitrag leistet; und dies bevor sichergestellt ist, daß auch der andere seinen Beitrag geleistet hat. Der für das Entstehen einer Kooperation notwendige Vertrauensvorschuß beruht dabei auf Gegenseitigkeit; alle sind hier gleichgestellt. Nur wo ohne abgesicherter Sanktionsmechanismen dieses Vertrauen und eigene Selbstbindung entsteht, da entstehen Kooperationen im eigentlichen Sinne.

Die Notwendigkeit des Vertrauens ist nun keineswegs als Nachteil der Kooperation zu verstehen, den es abzuschaffen gilt. Sie ist vielmehr ausgewiesenes Wesensmerkmal der Kooperation. Und sie ist verantwortlich dafiur, daß das Instrument der Kooperation weit weniger oft eingesetzt wird, als es Situationen gibt, in denen es effizient der Zielerreichung verschiedener potentieller Kooperationspartner eingesetzt werden könnte. Dies um so mehr, als in Kooperationssituationen, gekennzeichnet nun gerade auch durch das Fehlen unmittelbarer Sanktionspotentiale, zumindest nach mittelbaren Sanktionspotentialen gesucht werden kann. Damit kann die Bereitschaft zur Selbstbindung der Kooperationspartner, wenn schon nicht erzwungen, so doch zumindest gefördert werden.

Bei den im Umweltschutz so beliebten Selbstverpflichtungsabkommen sind diese Möglichkeiten übrigens gerade denkbar gering ausgeprägt. Zum einen bestehen aufgrund der großen Anzahl der Kooperationspartner nur geringe Kontrollmöglichkeiten, um Kooperationsbrïche überhaupt aufzudecken. Das Wissen darum fördert bekanntermaßen nicht gerade die Selbstbindung, sondern eher dessen Gegenteil, das Trittbrettfahren. Zum anderen werden bei Selbstverpflichtungsabkommen regelmäßig öffentliche Güter (z.B. nicht erlassene Gesetzesvorschriften) erstellt. Selbst im Falle eines Entdecktwerdens ist der Ausschluß aus dem Genuß eines solchen Gutes als Sanktion nicht möglich. Dies ist bei kollektiven Gütern anders, die tatsächlich nur denen zur Verfügung gestellt werden, die sich an ihrer Entstehung beteiligt haben (z.B. kooperativer Informationsaustausch). So gibt es zahlreiche andere Formen der Kooperation, die zum Teil wesentlich mehr Potential für Vertrauen und Selbstbindung bieten, die aber in der Diskussion um das Kooperationsprinzip im Umweltschutz eine untergeordnete Rolle spielen. Auf diese verschiedenen Erscheinungsformen soll nun eingegangen werden.

\section{Die Vielfalt möglicher Kooperationsformen}

Um die zahlreichen und sehr unterschiedlichen Erscheinungsformen der Unternehmenskooperation etwaś transparenter zu machen, können einige „Dimensionen der Kooperation“ genutzt werden. $\mathrm{Zu}$ nennen sind dazu etwa die 
Anzahl der an einer Kooperation Beteiligten, die Richtung der Kooperation, der Gegenstand der Kooperation, die räumliche Ausdehnung, die Offenheit für neue Mitglieder etc. Einen Überblick über mögliche Dimensionen und deren Ausprägungen bietet der Morphologische Kasten in der Abbildung.auf dieser Seite. Das dort aufgeführte Beispiel (A) kennzeichnet eine Kooperation etwa zwischen einem Autohersteller und einem Elektronikunternehmen durch Gründung eines Gemeinschaftsunternehmens zur Entwicklung und zum Vertrieb eines Hybridfahrzeugs. Das Beispiel (B) kennzeichnet ein branchenweites Selbstverpflichtungsabkommen etwa zum Verzicht auf die Verwendung von FCKW in Kïhlanlagen.

Auf eine der im Morphologischen Kasten aufgeführten Dimensionen soll hier besonders hingewiesen werden, da sie zur Klassifizierung von Kooperationen sehr wichtig scheint, aber bisher wenig verwendet wird. Dabei handelt es sich um die vier möglichen Ausprägungen der Vorgehensweise (3).

- Beim gemeinsamen Handeln erfolgt die Kooperation über die gemeinsame Durchführung von Aktivitäten unter Zusammenführung von Ressourcen. Typisch, aber nicht zwingend erforderlich dafiir ist die Ausgliederung von Teilfunktionen aus den kooperierenden Unternehmen in eine dafür geschaffene eigene Organisation wie z.B. ein separates Wirtschaftsunternehmen (siehe Beispiel A) oder einen Verband (z.B. Umweltverbände wie future, B.A.U.M. oder UnternehmensGrün). Gemeinsames Handeln kann aber auch auf weit geringeren Intensitätsstufen vorliegen, etwa durch das gemeinsame Betreiben eines Messestandes (z.B. Daimler Benz und Mitshubishi bei der Umweltausstellung „New Earth 93“ in 0saka).

- Beim abgestimmten Handeln agiert jeder Kooperationspartner weiterhin eigenständig. Allerdings werden die Handlungsfreiräume der Kooperationspartner verpflichtend untereinander abgestimmt. Beispiel dafür ist etwa der Aufbau eines Recyclingkreislaufs, in welchem sich einzelne Firmen zur Wahrnehmung bestimmter Teilaufgaben verpflichten. Aber auch Konzepte zur City-Logistik, also der Bündelung von Lieferverkehr in Innenstädten, basieren zum Teil auf solchermaßen abgestimmtes Handeln.

- Verbaltensabsprachen beinhalten alleine die Beschneidung vorhandener Freiräume. Es wird damit nicht die Durchführung bestimmter Tätigkeiten vereinbart, sondern alleine die Unterlas-

\section{Ökologie in der Ökonomie}

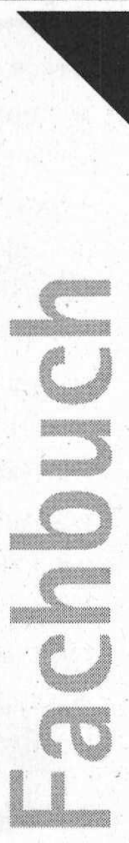

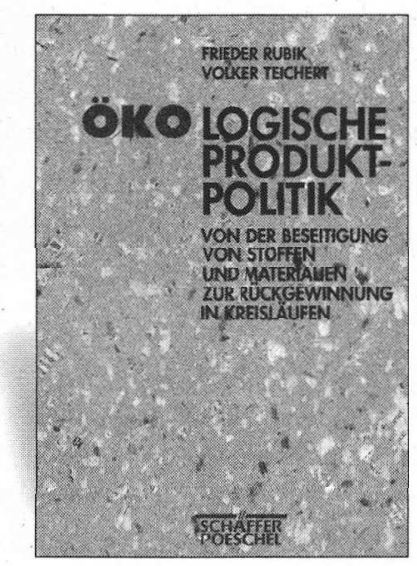

\section{F. Rubik/V. Teichert}

\section{Ökologische Produktpolitik}

Von der Beseitigung von Stoffen und Materialien zur Rückgewinnung in Kreisläufen

1997. 474 S. Kart., DM 78,-

ISBN 3-7910-0638-X

Mit Hilfe der Produktlinienanalyse wird Licht in die Behauptung von Unternehmen gebracht, ihre Produkte seien ökologisch unbedenklich hergestellt, vermarktet, verwertet, entsorgt. Der Verbraucher muß dies glauben oder nicht. Jetzt gibt es ein umweltpolitisches Instrumentarium von unbestechlicher Aussagekraft.

\section{S. Schaltegger/A. Sturm} Öko-Effizienz durch Öko-Controlling Zur praktischen Umsetzung von EMAS und ISO 14.001 1996. 176 S. Geb., DM 68,ISBN 3-7910-0992-3 Mit Hilfe von Praxisbeispielen wird gezeigt, wie das Öko-Controlling sowohl in der Produktion von Gütern als auch von Dienstleistungen wirkt, welche Informationen dazu notwendig sind, um z. B. Schadschöpfungskennzahlen oder Stoffund Energieflüsse zu berechnen oder zu einem Öko-Effizienz-Portfolio zu kommen und auch für kleinere und mittlere Unternehmen möglich ist. Hierfür waren sowohl die Flumroc AG als auch die Mohndruck AG bereit, ihre betrieblichen Daten zu veröffentlichen.

\section{Schäffer-Poeschel Verlạg}

Postfach 103241

70028 Stuttgart

Tel. (07 11) 2194-0

Fax (07 11) 21 94-119

http://www.schaeffer-poesschel.de

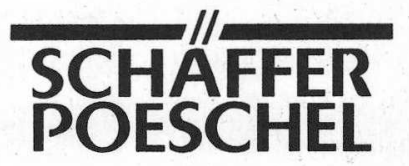


sung evtl. bisher durchgeführter Tätigkeiten. Typisches Beispiel für Absprachen sind Selbstverpflichtungsabkommen (siehe Beispiel B).

- Kooperativer Informationsaustausch bezeichnet die ggf. auch einseitige Überlassung von Informationen an andere Unternehmen, ohne daß aus der Verwendung dieser Informationen zwangsläufig Vorteile für das Unternehmen entstehen müssen, das die Informationen zur Verfuigung stellt. Es geht also nicht um einen Tausch, bei dem dank gleichartigen Leistungsinhalts auf das Zwischenmedium Geld verzichtet wird. Es geht vielmehr um Aufbereitung und Weitergabe von Informationen an andere Unternehmen, die diese für ihre Arbeit benötigen. Natürlich geht es nicht um die Weitergabe von Informationen, die gegen das Unternehmen eingesetzt werden sollen, das die Informationen liefert.

\section{Die Besonderheit der Netzwerke}

Nach dieser Strukturierung von Unternehmenskooperationen soll nun auf ein verwandtes Phänomen, nämlich auf das der Unternehmensnetzwerke eingegangen werden. Ziel ist es, eine inhaltliche Abgrenzung beider Begriffe vorzunehmen. Die Netzwerkforschung, um die sich in jüngerer Zeit zahlreiche einzelne Forscher (4) wie auch Forschungsgruppen (5) verdient gemacht haben, fokussiert in ihren Bemühungen nämlich primär auf eine Form von Netzwerken, die nicht unmittelbar aus den gerade beschriebenen Dimensionen der Kooperation hergeleitet werden kann. Netzwerke werden dort selten als Ergebnis nur einer spezifischen Kooperationsvereinbarung diskutiert (z.B. ein „Netzwerk für Umweltberatung“), sondern zumeist als das Ergebnis einer Vielzahl sich überlagernder Kooperationen. Einzelne Unternehmen innerhalb eines Netzwerkes sind aber an mehreren Kooperationen beteiligt (vgl. die Abbildungen auf Seite 14). Damit sind die Grenzen eines Netzwerkes auch selten so scharf zu ziehen, wie es bei einzelnen Kooperationen möglich ist. Die Teilnehmer der Kooperation, aus deren Überlagerung sich das Netzwerk ergibt, müssen nämlich keineswegs auf eine bestimmte Gruppe von Unternehmen begrenzt sein.

Der damit attestierte, eher mittelbare Entstehungscharakter von Netzwerken bedeutet aber nicht, daß Netzwerke nicht strategisch angestrebt und aufgebaut werden könnten. Der Weg dahin führt aber zunächst über die Bil- dung einzelner Kooperationen, die dann in ihrer Gesamtheit das angestrebte Netzwerk ergeben.

\section{Kooperationsstrafegien als Zielerreichungsstrategien}

Kooperationen (und natürlich Netzwerke), wie sie hier definiert und dargestellt wurden, können strategisch genutzt werden, um die zur Erreichung bestimmter Ziele erforderlichen Ressourcen aufzubauen. Entsprechende Kooperationsstrategien befinden sich in der Gesellschaft von Autonomie- und Beteiligungsstrategien. Mit diesen wird versucht, strategisch benötigte Ressourcen durch die Weiterentwicklung eigener, bereits vorhandener Potentiale aufzubauen oder diese Ressourcen einzukaufen (zum Teil in Form ganzer Unternehmen).

Kooperationsstrategien sind nun keineswegs als per se ökologisch richtigere Strategien zu bevorzugen. Alle drei Strategieformen haben in einer Marktwirtschaft ihre volle Berechtigung. Kooperationsstrategien können aber zur einzig möglichen Strategievariante werden, wenn eigene Potentiale zur Weiterentwicklung nicht vorhanden und die Voraussetzungen für klassische Kaufverträge nicht herzustellen sind. In dieser Situation heißt die Alternative dann nicht mehr: Kooperations- oder Autonomieoder Beteiligungsstrategie, sondern: Kooperationsstrategie oder Verzicht auf Zielverfolgung. Dies ist eine Rahmenbedingung, die für viele ökologische Ziele durchaus typisch ist. Man denke etwa an das Ziel der Verbesserung ökologischer Produktlebenszyklen. Die dazu notwendigen Informationen über vor- und nachgelagerte Produktions- und Transportstufen lassen sich nicht am Markt kaufen. Sie müssen von den beteiligten Unternehmen selbst ermittelt und weitergereicht bzw. ausgetauscht werden. Entweder es gelingt hier, eine Kooperation aufzubauen, oder das Ziel der Verbesserung des ökologischen Produktlebenszyklus kann nicht verfolgt werden.

Der strategische Einsatz von Kooperationen gehört allerdings (noch) keineswegs zum geübten Standardrepertoire westlich geprägten Wirtschaftshandelns. Der hierzu notwendige Vertrauensvorschuß gegenüber strategischen Kooperationspartnern und die notwendige Selbstbindung vertragen sich scheinbar zu wenig mit den Vorstellungen einer Marktwirtschaft, deren idealisierte Basis die vollständige Konkurrenz ist. Es ist jedoch wenig lebensnah (und damit auch wissenschaftlich wenig nützlich), Markt als Ort oder Synonym der Konkurrenz zu setzen. Eine Marktwirtschaft benötigt zwar unbedingt Konkurrenz, mit der bestimmte Ziele nur erreicht werden, wenn andere Ziele nicht erreicht werden. Sie benötigt aber ebenso Kaufverträge, mit denen alle Vertragspartner ihre Ziele erreichen. Und sie benötigt und lebt von Kooperationen, mit denen die Beteiligten diese Ziele nur erreichen können, wenn sie sich vertrauen und sich selbst binden. Kooperationen sind aufgrund dieser Voraussetzung eine sehr eigenständige Form der Koordination. Vielleicht werden sie deshalb noch bei weitem nicht in dem Maße genutzt, wie es für ökologische Veränderungen des Wirtschaftens erforderlich und auch möglich wäre.

Kooperation in dem hier dargestellten Sinne sollte aber nicht als etwas normativ besonders Wünschenswertes begriffen werden. Sie sollte als eine von drei möglichen Strategievarianten begriffen werden, die es ermöglicht, bestimmte unternehmerische Ziele sehr effizient zu erreichen und manche - zum Beispiel einige ökologische Ziele - überhaupt erst zu verfolgen.

\section{Anmerkungen}

1) Vgl. Deutsch, M. (1949): A theory of Co-operation and competition. In: Human Relations 2, S. 129-152.

2) Biesecker, A. (1996): Kooperation, Netzwerk, Selbstorganisation - Prinzipien für eine faire und vorsorgende Ökonomie. In: Biesecker, A./Grenzdörffer, K. (Hg.): Kooperation, Netzwerk, Selbstorganisation. Elemente demokratischen Wirtschaftens. S. 9-21.

3) Vgl. ausführlicher zu Erscheinungsformen der Kooperation Aulinger, A. (1996): (Ko-)Operation Ökologie. Kooperationen im Rahmen ökologischer Unternehmenspolitik. Marburg. S. $69 f f$.

4) Im deutschsprachigen Raum ist hier sicher Sydow hervorzuheben; vgl. Sydow, J. (1993): Strategische Netzwerke. Evolution und Organisation. 2. Aufl., Wiesbaden. 5) Aus der Forschergruppe um Håkansson ging sogar ein eigener Theorieansatz für Netzwerke, der "Netzwerkansatz" hervor; vgl. Håkansson, H. (Hg. 1987): Industrial Technological Development: A Network Approach. Croom Helm. London etc.; Håkansson, H. (1989): Corporate Technological Behaviour. Co-operation and Networks. London, New York.

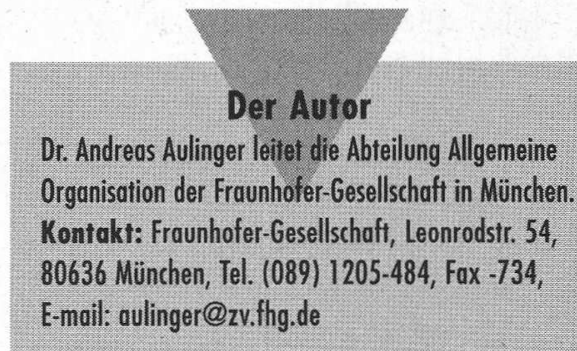


(c) 20I0 Authors; licensee IÖW and oekom verlag. This is an article distributed under the terms of the Creative Commons Attribution Non-Commercial No Derivates License (http://creativecommons.org/licenses/by-nc-nd/3.o/), which permits unrestricted use, distribution, and reproduction in any medium, provided the original work is properly cited. 